\title{
Interaction between sympathetic nervous system and renin angiotensin system on MMPs expression in juvenile rat aorta
}

\author{
Houcine Dab ${ }^{1,2}$, Rafik Hachani ${ }^{1}$, Wassim Hodroj ${ }^{2}$, Mohsen Sakly ${ }^{1}$, Giampiero Bricca ${ }^{2}$ \\ and Kamel Kacem ${ }^{1}$ \\ ${ }^{1}$ Laboratoire de Pathologies Vasculaires, Unité de Physiologie Intégrée, Faculté des Sciences de Bizerte, Université de Carthage, Tunisia \\ ${ }^{2}$ INSERM ERI-22, Agressions Vasculaires et Réponses Tissulaires, Université Claude Bernard - Lyon 1, France
}

\begin{abstract}
The aim of our present study is to investigate the interaction between angiotensin II (ANG II) and sympathetic nervous system (SNS) on matrix metalloproteinase MMP-2 and MMP-9 expression and activity in juvenile rat aorta under normal conditions. Sympathectomy with guanethidine and blockade of the ANG II receptors (AT1R) by losartan were performed alone or in combination on new-born rats. mRNA, protein expression and activity of MMP-2 and MMP-9 were examined by Q-RT-PCR, immunoblotting and zymography, respectively.

MMP-2 mRNA and protein amount were decreased after sympathectomy or AT1R blockade and an additive effect was observed after combined treatment. However, MMP-9 expression was reduced to the same level in the three treated groups. There were some detectable gelatinolytic activity of the MMPs in both control and treated rats.

We concluded that ANG II stimulates directly and indirectly (via sympathostimulator pathway) the MMP-2 expression but seems unable to affect MMP-9 expression through direct pathway. Combined inhibition of SNS and ANG II were more efficient than a single inhibition in reducing MMP amounts in rat vessels.
\end{abstract}

Key words: Sympathectomy - Angiotensin II - AT1R antagonist - MMP-2 - MMP-9 - Aorta

\section{Introduction}

Matrix metalloproteinases (MMPs) represent a tightly regulated family of zinc-dependent endopeptidases that degrade most components of the extracellular matrix (ECM) and basement membrane (Nagase and Brew 2003). Most MMPs are synthesized and secreted as inactive proenzymes and require proteolytic activation principally by plasmine and other MMPs to be functional in tissue and regulated by the tissue inhibitors of MMPs (TIMP) (Messerli 2004). Among the members of MMPs family, MMP-2 and MMP-9 have gained lots of attention since they are expressed in cardiovascular system and contribute to the degradation of several ECM proteins such as elastin and collagen (Galis and Khatri 2002). MMPs are largely implicated in ECM turn-over regulation

Correspondence to: Houcine Dab, Laboratoire de Pathologies Vasculaires, Unité de Physiologie Intégrée, Faculté des Sciences de Bizerte, Université de Carthage, Jarzouna 7021, Tunisia E-mail: houcine.dab@fsb.rnu.tn with contribution of other factors such as neuroendocrine systems especially the renin angiotensin system (RAS) and the sympathetic nervous system (SNS) (Miner and Miller 2006). Action of SNS and angiotensin II (ANG II) on MMPs expression and activity on heart tissue were well described in our previous study (Dab et al. 2009) and in another in vivo and in vitro studies which demonstrated that ANG II increases MMP- 2 content and activity in myocyte (Coker et al. 2001). Thus, an interaction between the SNS and ANG II has been described principally by the ANG II-induced stimulation of norepinephrine release from SNS through ANG II receptors (AT1R) expressed on presynaptic SNS terminal axon (Balt et al. 2001). On the other hand, the blockage of AT1R lowers the norepinephrine releases (Dendorfer et al. 1998) and enhances its uptake by SNS terminal axon (Raasch et al. 2004). However, the interaction between SNS and RAS on MMPs expression and activity is not well defined in vascular system under normal conditions.

The aim of this study is to investigate the interactions of the SNS and AT1R-mediated angiotensin effects on MMPs 
expression and activity in the aorta of rat. The experimental protocol was conducted by performing sympathectomy and blockade of AT1R with losartan alone or in combination. For this reasons, mRNA, protein and enzymatic activity of MMP-2 and MMP-9 were determined by RT-PCR, immunoblotting and zymography methods in the aorta of juvenile normotensive rats.

\section{Materials and Methods}

\section{Animals}

The animal protocols used for this study were approved by the University Animal Care and Use Committee of Claude Bernard University in France and were in accordance with the National Institutes of Health Guidelines for the Care and Use of Laboratory Animals.

New-born Wistar-Kyoto rats $(n=40)$, were equally divided into four groups: a sympathectomy group, a losartantreated group, a sympathectomy and losartan-treated group and a sham group. One week aged rats were sympathectomized with guanethidine sulphate injection $(50 \mathrm{mg} / \mathrm{kg}$ i.p.; 5 days/week) for 3 weeks (Johnson et al. 1976). The success of guanethidine injections was approved by the development of ptosis (Dwyer et al. 2004). The losartan-treated group received $20 \mathrm{mg} / \mathrm{kg} /$ day of losartan (Sigma, St. Louis, USA), in drinking water (Rocha et al. 2007) until 6 weeks of age. The water consumption and the rat body weight were measured daily and the amount of losartan was adjusted accordingly. The sham group received injections of saline for the same period of time as for the sympathectomy group.

At 6 weeks of age, the rats were deeply anesthetized with pentobarbital, the aorta was removed, washed from blood, then frozen immediately in liquid nitrogen, and stored at $-80^{\circ} \mathrm{C}$.

\section{Total RNA extraction and real-time RT-PCR analysis}

RNA extraction and real-time RT-PCR analysis are described in our previous study (Dab et al. 2009). Briefly, total RNA was extracted from the aorta using Trizol Reagent (Invitrogen, Carlsbad, USA). After DNase treatment, $2 \mu \mathrm{g}$ of total RNA was reverse-transcripted with superscript II transcriptase (Invitrogen, France) using random hexamers as primers (pdN6; Amersham Biosciences, Piscataway, USA). Quantitative real-time RT-PCR was performed for selected gene transcripts with a MyiQ thermal cycler (BioRad Laboratories, Hercules, USA). Real-time RT-PCR was performed using the resulting cDNA as template, iQ SYBR Green Supermix (Bio-Rad Laboratories, Hercules, USA), and the appropriate set of primers (Invitrogen, France) specific to MMP-2 (sense: ACGATGGCAAGGTGTGGTGT; antisense: CCTTGGTCAGGACAGAAGCC), MMP-9 (sense: CGAGCTATCCACTCATCAAACAT; anti-sense: GTGTCCTCCGATGTAAGAGAGAA). 18S ribosomal RNA (18S rRNA) are used as housekeeping gene internal controls (sense: AGTCGGCATCGTTTATGGTC; anti-sense: TGAGGCCATGATTAAGAGGG). Cycle threshold values were calculated for the different products using Optical System Software version 1.0 (Bio-Rad Laboratories, Hercules, USA) PCR was performed in duplicate.

\section{Protein extraction and Western blotting}

The protocol of protein extraction and immunoblotting was previously described (Dab et al. 2009). Briefly, Frozen aortic tissue $(100 \mathrm{mg}$ ) was homogenized in hydrolysis buffer containing protease inhibitor cocktail (Complete mini, Roche, $\mathrm{Mu}-$ nich, Germany). The protein concentrations were measured in the supernatants with the BCA kit (Pierce, USA). $50 \mu \mathrm{g}$ of protein extract were loaded on to a $10 \%$ sodium dodecyl sulfate-polyacrylamide gel electrophoresis (SDS-PAGE). The separated proteins were transferred to nitrocellulose membranes through a transfer medium. After blockage with Tris-buffered saline Tween-20 (TBST), 0.1\% (v/v), containing powdered goat milk, membranes were incubated overnight at $4^{\circ} \mathrm{C}$ with specific mouse monoclonal IgG antibodies to MMP-2 and MMP-9 $(1: 1000)$ or with monoclonal IgG anti- $\beta$-actin ( 1 : 5000). After washing with $0.1 \%$ TBST, membranes were incubated with the horseradish peroxidase-conjugated goat anti-mouse IgG antibody $(1: 5000)$ for $1 \mathrm{~h}$. All antibodies were purchased from Sigma (Sigma, St. Louis, USA). Immunoreactivity of the proteins was visualized by chemiluminescent detection using an enhanced chemiluminescent reagent (ECL, Amersham, Buckinghamshire, UK).

\section{MMPs zymography}

Frozen aortic tissue were pulverized in liquid nitrogen and then homogenized in cacodylic acid at $\mathrm{pH} 5,150 \mathrm{mM} \mathrm{NaCl}$, $1 \mathrm{mM} \mathrm{ZnCl}_{2}, 20 \mathrm{mM} \mathrm{CaCl}_{2}, 1.5 \mathrm{mM} \mathrm{NaN}_{3}$, and $0.01 \%$ (vol/ vol) Triton $\mathrm{X}-100)$ at $4^{\circ} \mathrm{C}$, after extraction the $\mathrm{pH}$ was raised by the addition of $1.5 \mathrm{M}$ Tris $\mathrm{HCl}(\mathrm{pH} 8.8)$. Aortic extracts containing $50 \mu \mathrm{g}$ of proteins were analysed by electrophoresis on $7.5 \%$ SDS-PAGE containing $1 \mathrm{mg} / \mathrm{ml}$ of porcine skin gelatin (Sigma) under non-denaturing conditions (Dab et al. 2009). After electrophoresis, the gels were first washed twice in $2.5 \%$ Triton X-100 for 30 minutes to remove SDS and to allow the proteins renaturation, then rinsed in water and incubated for $48 \mathrm{~h}$ in a substrate buffer at $37^{\circ} \mathrm{C}(50 \mathrm{mM}$ Tris- $\mathrm{HCl}, 5 \mathrm{mM} \mathrm{CaCl}_{2}$, and $0.02 \% \mathrm{NaN}_{3}, \mathrm{pH} 7.4$ ). At the end of the incubation, gels were stained with Coomassie Blue R- 
250 for $1 \mathrm{~h}$, then transferred to a $10 \%$ acetic acid and $40 \%$ methanol solution for $1 \mathrm{~h}$ and then kept in $10 \%$ acetic acid solution. The active form of MMPs appears as areas of lysis on a dark blue Coomassie-stained gelatin background.

\section{Statistical analysis}

Statistical comparisons between treated and control groups were performed by variance analysis (ANOVA). Subsequent pair-wise comparisons were performed with the Tukey HSD test using statistical software (Statsoft, France). Results are expressed as mean \pm SEM. $p<0.05$ is taken as a significant probability and $n$ values indicate the number of experiments.

\section{Results}

\section{Local expression of MMPs}

In the sympathectomy group (Gua), MMP-2 and MMP-9 mRNA expressions were decreased significantly by $19 \%$ and $21 \%$, respectively. In the losartan-treated group (Los), MMP-2 and MMP-9 mRNA expressions were similarly modified and decreased significantly by $14 \%(p<0.05)$. MMP-9 mRNA expression was similar in combined and single treatments, while MMP-2 expression was strongly decreased by $31 \%(p<0.01)$ versus control (Fig. 1$)$. mRNA of MMP- 9 was similar in the three treated groups.

\section{口MMP-2 DMMP-9}

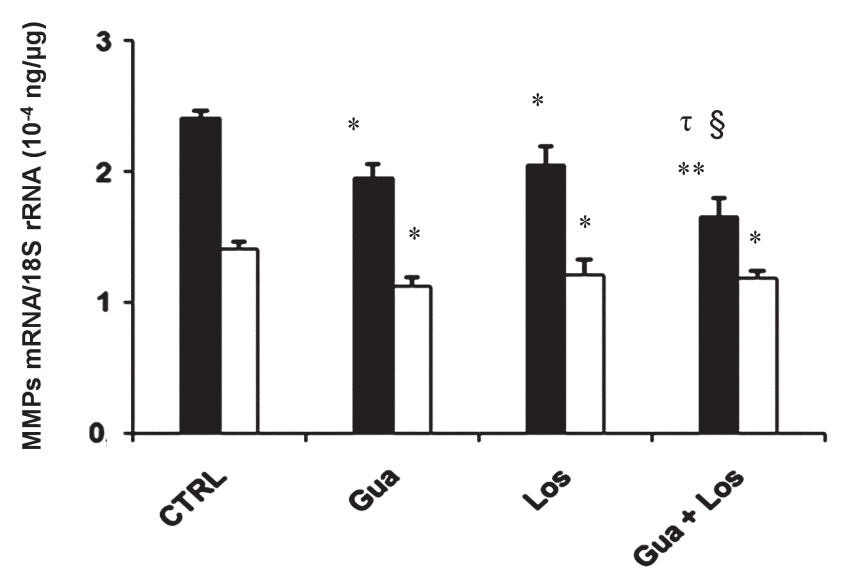

Figure 1. Quantitative real-time RT-PCR of MMP-2 and MMP-9 mRNA in aorta from control and treated rats. Values are presented as ratio of mRNA to $18 \mathrm{~S}$ rRNA. Data are mean \pm SEM; $n=10$ in each group. ${ }^{\star} p<0.05,{ }^{* *} p<0.01$ vs. CTRL, ${ }^{\tau} p<0.05$ vs. sympathectomy group, ${ }^{\S} p<0.05$ vs. losartan-treated group. CTRL, control group; Gua, sympathectomy group; Los, losartan-treated group; Gua + Los, combined treatment group.
The evolution of MMPs protein expression after the three treatments was similar to the mRNA expression. In the Gua group, MMP-2 and MMP-9 protein expressions decreased significantly by $11 \%(p<0.05)$ and $35 \%(p<0.05)$, respectively. In the Los group, MMP-2 and MMP-9 expressions were decreased significantly by $15 \%(p<0.05)$ and $40 \%(p<$ $0.05)$, respectively. After combined treatment only MMP-2 protein expression was significantly decreased by $29 \%(p<$ 0.01) (Fig. 2).

\section{MMPs activity}

Any gelatinolytic activity of MMPs was detected in aortic samples from both control and treated rats.

\section{Discussion}

The aim of the present study is to evaluate the contribution of SNS and ANG II and interaction between the two systems on MMPs expression and activity in rat aorta under normal conditions and based on our previous investigation in heart (Dab et al. 2009). Distinction between the two pathways of ANG II action was assisted by performing sympathectomy and AT1R blockade separately or in combination in normotensive rats.

In this study the use of single or combined inhibition of SNS and ANG II allows a better differentiation of the direct and indirect action of ANG II through AT1R expressed in SNS axons. These experimental protocol leads to compare single inhibition of the two systems which is widely used to reduce cardiovascular mortality and morbidity and to investigate the effects of combined inhibition and combined treatment on MMPs expression and activity.

Guanethidine destroys cells bodies of sympathetic ganglia and produces permanent terminal axons degeneration that reached the blood vessels and the heart (Heath and Burnstock 1977). Guanethidine induces also an important loss of norepinephrine amount in circulation and in all tissues (Villanuvea et al. 2003) except that of the adrenal gland. In the present study, the manifestation of ptosis approves the efficiency of guanethidine treatment in SNS destroying (Supowit and al. 2005).

All conclusions about the effect of ANG II through direct and indirect (via SNS) are deduced from our experimental approach. We have schematized our conclusion in Fig. 3 to better explain the interaction between the SNS and the ANG II. As represented in this figure, AT1R is expressed in sympathetic fibres coursing blood vessels (presynaptic receptors) and in arterial cells. Based on our previous publications, our hypothesis was that ANG II from circulation or locally synthesised, influence the local synthesis of MMPs by two pathways: The first pathway is indirect and it is by sympa- 
A

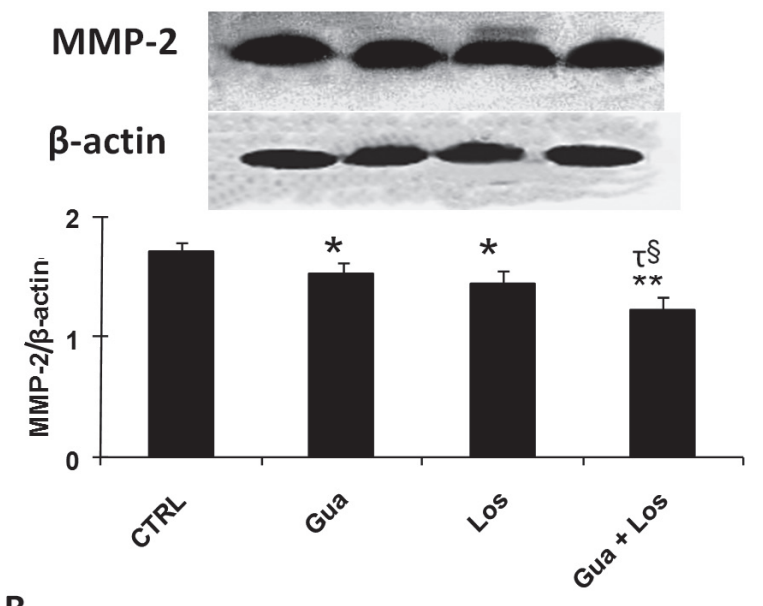

B

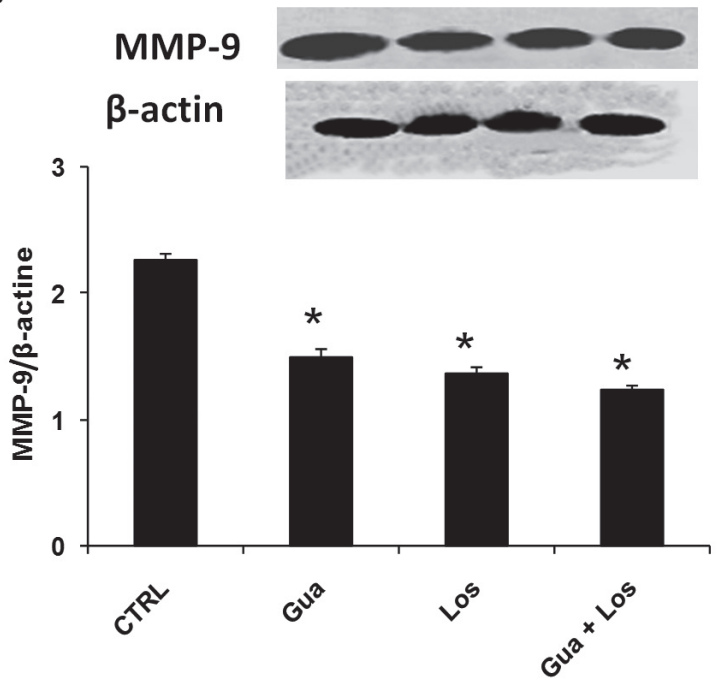

Figure 2. Immunoblot analysis and densitometric quantification of MMP-2 (A) and MMP-9 (B) in aorta from control and treated rats. Values are presented as ratio of MMP- 2 or MMP- 9 content to $\beta$-actin. Data are shown as mean values \pm SEM; $n=10$ in each group. ${ }^{\star} p<0.05,{ }^{* *} p<0.01$ vs. CTRL, ${ }^{\tau} p<0.05$ versus sympathectomy group, ${ }^{\S} p<0.05 v s$. losartan-treated group. CTRL, control group; Gua, sympathectomy group; Los, losartan-treated group; Gua + Los, combined treatment group.

thostimulator where ANG II enhance neurotransmitters release from sympathetic fibres including norepinephrine (Gironacci et al. 1994; Dendorfer et al. 1998; Balt et al. 2001; Raasch et al. 2004). Norepinephrine acts through noradrenergic receptors expressed on aortic cells. The second pathway is a direct one where ANG II mediates its action on aortic tissue via AT1R.

To identify the part of each way of ANG II, we proposed that 1) in the Gua group, sympathectomy induces elimina- tion of SNS and consequently the sympathostimulator pathway of ANG II. In this case, ANG II remains freely accessible only to AT1R expressed in aortic tissue (only direct pathway was functional). 2) In the group Gua+Los the combination of sympathectomy and AT1R blockade with losartan leads to abolish both direct and indirect pathway of ANG II, and therfore, the comparison between Gua and Gua+Los allows an examination of ANG II action mediated via its AT1R through direct pathway. 3) In the Los group, losartan induces blockage of AT1R in both presynaptic sympathetic fibres and aortic cells tissue. In this group the norpinephrine remains accessible to aortic tissue. Trophic action of SNS that reflects the action of ANG II through indirect pathway can be deducted by comparing groups Los and Gua+Los.

\section{MMP-9 expression}

When the AT1R in aortic tissue were free (in the Gua group) or blocked (in the Gua+Los group), mRNA and protein expression of MMP-9 were reduced by the same order excluding thus the intervention of ANG II via AT1R expressed in aortic cells (direct pathway). The fall in MMP-9 expression is due to either complete elimination of SNS by sympathectomy (Gua and Gua+Los groups) or to the decrease of norepinephrine releases from sympathetic fibers induced by blockage of AT1 presynaptic receptor. In this case, ANG II mediates stimulation only through sympathostimulator pathway.

\section{MMP-2 expression}

When we used the same analysis of results like MMP-9, we observed that mRNA and protein expression of MMP-2 was more inhibited after sympathectomy when AT1R are blocked (Gua+Los group) and free (Gua group), so we can conclude that ANG II enhances MMP-2 expression by direct pathway. The fall of MMP-2 expression in the Gua+Los compared to Los group reveals that ANG II is a stimulator by sympathostimulator pathway.

Stimulatory action of SNS is mediated by the secretion of norepinephrine on the vascular wall as shown in literature. Therefore MMPs expression is enhanced in human atherosclerotic plaques (Sukhova et al. 1999) and the direct action of ANG II on MMPs secretion may be potentiated by SNS, cytokines and chemokines (Qin 2008). In vitro studies showed that ANG II enhances mRNA synthesis and activity of MMP-2 in murine smooth muscle cells (Luchtefeld et al. 2005). Ex vivo studies also demonstrated that ANG II increase MMP2 activity in carotid rings from young rats abolished by losartan (Wang et al. 2005).

In addition, norepinephrine-induced MMPs expression by nasopharyngeal carcinoma through $\beta$-adrenergic receptors (Yang et al. 2006) and the use of $\alpha$ and $\beta$ adrenergic receptors decreases MMP-2 and MMP-9 expression on rat 
aortic wall (Wu et al. 2007). Norepinephrine-associated upregulated MMPs expression can be caused by an up-regulated proinflammatory cytokines production, such as interleukin IL-1, TNF- $\alpha$, and IL-6.

ANG II enhances MMPs expression by human smooth muscle cells by activation of nuclear factor $-\kappa B$ and MMP-1, MMP-3 and MMP-9 by aortic smooth muscle cells and can be prevented by losartan (Browatzki et al. 2005, Guo et al. 2008). ANG II also increases MMP-2 and MMP-9 amount in aneurismal aorta (Eagleton et al. 2006).

\section{MMPs activity}

We are unable to deduce from the present results the effect of SNS and ANG II and SNS on MMPs activity since any gelatinolytic activity was observed in aorta. The lack of MMPs activity can be explained by a lower amount of protein of each MMPs in studied tissues as it was previously mentioned (Lindsey et al. 2001).

There exist limited information about the regulation of MMPs expression and activity by SNS and ANG II in vivo and under normal conditions. In this perspective, in our previous investigation (Dab et al. 2009) in left ventricle, we established that ANG II seems unable to influence MMPs activities directly via AT1R, since the activities were not modified when AT1R were free or blocked by losartan; however, SNS acts as stimulator since MMPs activities were reduced after sympathectomy. On the other hand, it has been demonstrated that blockade of AT1R by irbesartan modulate MMPs activity in carotid artery stenosis (Cipollone et al. 2004), this disparity in ANG II action seems to be caused by the difference between the used tissues and also in the difference between normal and pathological conditions in the two studies.

In this study, the lack of enzymatic activity makes unclear the effect of each system on MMPs activity. But the examination of previous investigation on MMPs expression and activity indicates that enzymatic activities of these enzymes are strongly linked to the protein and/or mRNA expression and the balance between activators and inhibitors (Wang et al. 2005). Thus, it has been demonstrated that both the protein amount and activity of each MMPs are regulated by the same signal transduction pathway. Therefore, MMP-9 expression and activity were enhanced by the activation of c-Jun $\mathrm{NH}_{2}$-terminal kinase (JNKs) through protein kinase $\mathrm{C}$ (PKC) $-\alpha / \beta 1$. In addition, the expression and activity of both MMP- 2 and MMP-9 are regulated by PKC- $\theta$ and $\mathrm{PKC}-\zeta$ by activation of JNKs, extracellular signal-regulated kinase (ERK)1/2, and NF- $\kappa B$ (Xie et al. 2004).

Acknowledgements. We wish to thank the "Autour de Williams" association for their financial participation to provide materials used in our experiments. Houcine Dab received a grant from the Tunisien Ministry of Higher Education, Scientific Research and Technology to work on this study in the EA4173, INSERM ERI-22 laboratory, Faculté Rockefeller, Université Claude Bernard, Lyon 1, France.

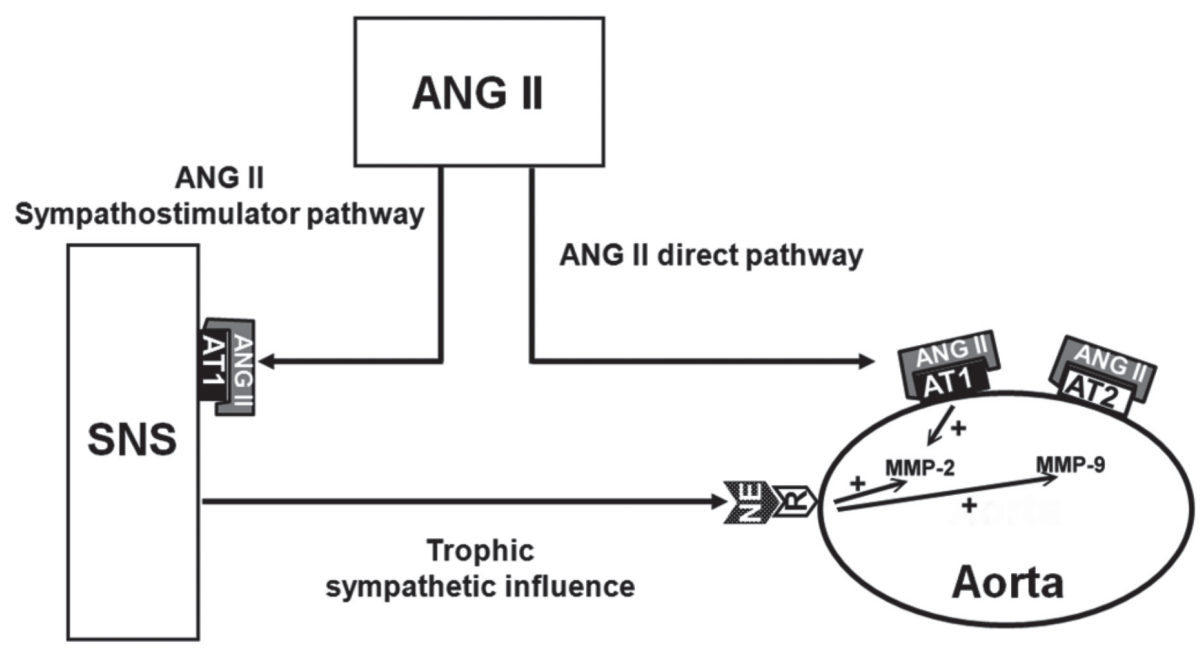

Figure 3. Schematic pathways of hypothetic neurohumoral regulation of endogenous ECM proteolysis in the heart. Both AT1 and AT2 receptors of ANG II exist in sympathetic fibers coursing the heart (presynaptic receptors) and in cardiac cells (Timmermans and Smith, 1994). Based on the literature, we hypothesise that Ang II from circulation or locally synthesized acts through two pathways to influence the local synthesis of t-PA, PAI-1, MMPs and ECM: 1) Sympathostimulator pathway where ANG II incites neurotransmitters release from sympathetic fibers including norepinephrine (Gironacci et al. 1994; Dendorfer et al. 1998; Balt et al. 2001; Raasch et al. 2004). Norepinephrine (NE) assure its action through noradrenergic receptors (R) expressed on cardiac cells; 2) direct pathway where ANG II mediates directly its action on cardiac tissue via AT1R essentially but can also access to AT2 receptor. Empty arrows: ANG II-mediated action indirectly via SNS or directly via AT1 receptors on MMPs expression; + stimulation. 


\section{References}

Balt J. C., Mathy M. J., Nap A., Pfaffendorf M., van Zwieten P. A. (2001): Effect of the AT1-receptor antagonists losartan, irbesartan, and telmisartan on angiotensin II-induced facilitation of sympathetic neurotransmission in the rat mesenteric artery. J. Cardiovasc. Pharmacol. 1, 141-148

doi:10.1097/00005344-200107000-00015

Browatzki M. D., Pfeiffer C. A., Gehrke S. G., Schmidt J., Kranzhofer A., Katus H. A. Kranzhofer R. (2005): Angiotensin II stimulates matrix metalloproteinase secretion in human vascular smooth muscle cells via nuclear factor-kappaB and activator protein 1 in a redox-sensitive manner. J. Vasc. Res. $5,415-423$ doi:10.1159/000087451

Cipollone F., Fazia M., Iezzi A., Pini B., Cuccurullo C., Zucchelli M., de Cesare D., Ucchino S., Spigonardo F., De Luca M., Muraro R., Bei R., Bucci M., Cuccurullo F., Mezzetti A. (2004): Blockade of the angiotensin II type 1 receptor stabilizes atherosclerotic plaques in humans by inhibiting prostaglandin E2-dependent matrix metalloproteinase activity. Circulation 109, $1482-1488$ doi:10.1161/01.CIR.0000121735.52471.AC

Coker M. L., Jolly J. R., Joffs C., Etoh T., Holder J. R., Bond B. R., Spinale F. G. (2001): Matrix metalloproteinase expression and activity in isolated myocytes after neurohormonal stimulation. Am. J. Physiol. Heart. Circ. Physiol. 2, H543-551

Dab H., Hachani R., Hodroj W., Sakly M., Bricca G., Kacem K. (2009): Differential control of MMP and t-PA/PAI-1 expressions by sympathetic and renin-angiotensin systems in rat left ventricle. Auton. Neurosci. 2, 27-32 doi:10.1016/j.autneu.2009.04.002

Dendorfer A., Raasch W., Tempel K., Dominiak P. (1998): Interactions between the renin-angiotensin system (RAS) and the sympathetic system. Basic. Res. Cardiol. 93, 24-29 doi:10.1007/s003950050202

Dwyer K. W., Provenzano P. P., Muir P., Valhmu W. B., Vanderby R. Jr. (2004): Blockade of the sy.mpathetic nervous system degrades ligament in a rat MCL model. J. Appl. Physiol. 96, $711-718$ doi:10.1152/japplphysiol.00307.2003

Eagleton M. J., Ballard N., Lynch E., Srivastava S. D., Upchurch G. R. Jr., Stanley J. C. (2006): Early increased MT1-MMP expression and late MMP-2 and MMP-9 activity during Angiotensin II induced aneurysm formation. J. Surg. Res. 2, 345-351 doi:10.1016/j.jss.2006.03.026

Galis Z. S., Khatri J. J. (2002): Matrix metalloproteinases in vascular remodeling and atherogenesis: the good, the bad, and the ugly. Circ. Res. 3, 251-262

Gironacci M. M., Adler-Graschinsky E., Peña C., Enero M. A. (1994): Effects of angiotensin II and angiotensin-(1-7) on the release of $[3 \mathrm{H}]$ norepinephrine from rat atria. Hypertension 24, 457-460

Guo R. W., Yang L. X., Wang H., Liu B., Wang L. (2008): Angiotensin II induces matrix metalloproteinase- 9 expression via a nuclear factor-kappaB-dependent pathway in vascular smooth muscle cells. Regul. Pept. 3, 37-44 doi:10.1016/j.regpep.2007.12.005
Heath J. W., Burnstock G. (1977): Selectivity of neuronal degeneration produced by chronic guanethidine treatment. J. Neurocytol. 4, 397-405 doi:10.1007/BF01178225

Johnson E. M. Jr., O’Brien F., Werbitt R. (1976): Modification and characterization of the permanent sympathectomy produced by the administration of guanethidine to newborn rats. Eur. J. Pharmacol. 1, 45-54 doi:10.1016/0014-2999(76)90006-6

Lindsey M., Wedin K., Brown M. D., Keller C., Evans A. J., Smolen J., Burns A. R., Rossen R. D., Michael L., Entman M. (2001): Matrix-dependent mechanism of neutrophilmediated release and activation of matrix metalloproteinase 9 in myocardial ischemia/reperfusion. Circulation 17, 2181-2187

Luchtefeld M., Grote K., Grothusen C., Bley S., Bandlow N., Selle T., Strüber M., Haverich A., Bavendiek U., Drexler H., Schieffer B. (2005): Angiotensin II induces MMP-2 in a p47phox-dependent manner. Biochem. Biophys. Res. Commun. 1, 183-188 doi:10.1016/j.bbrc.2004.12.152

Messerli F. H. (2004): TIMPs, MMPs and cardiovascular disease. Eur. Heart. J. 17, 1475-1476 doi:10.1016/j.ehj.2004.07.015

Miner E. C., Miller W. L. (2006): A look between the cardiomyocytes: the extracellular matrix in heart failure. Mayo. Clin. Proc. $1,71-76$ doi:10.4065/81.1.71

Nagase H., Brew K. (2003): Designing TIMP (tissue inhibitor of metalloproteinases) variants that are selective metalloproteinase inhibitors. Biochem. Soc. Symp. 70, 201-212

Qin Z. (2008): Newly developed angiotensin II-infused experimental models in vascular biology. Regul. Pept. 150, 1-6 doi:10.1016/j.regpep.2008.05.002

Raasch W., Dominiak P., Ziegler A., Dendorfer A. (2004): Reduction of vascular noradrenaline sensitivity by AT1 antagonists depends on functional sympathetic innervation. Hypertension 3, 346-3451 doi:10.1161/01.HYP.0000138406.13413.0e

Rocha F. L., Carmo E. C., Roque F. R., Hashimoto N. Y., Rossoni L. V., Frimm C., Anéas I., Negrão C. E., Krieger J. E., Oliveira E. M. (2007): Anabolic steroids induce cardiac renin-angiotensin system and impair the beneficial effects of aerobic training in rats. Am. J. Physiol. Heart. Circ. Physiol. 6, H3575-3583 doi:10.1152/ajpheart.01251.2006

Sukhova G. K., Schönbeck U., Rabkin E., Schoen F. J., Poole A. R., Billinghurst R. C., Libby P. (1999): Evidence for increased collagenolysis by interstitial collagenases- 1 and -3 in vulnerable human atheromatous plaques. Circulation 99, 2503-2509

Supowit S. C., Ethridge R. T., Zhao H., Katki K. A., Dipette D. J. (2005): Calcitonin gene-related peptide and substance $P$ contribute to reduced blood pressure in sympathectomized rats. Am. J. Physiol. Heart. Circ. Physiol. 3, H1169-1175 doi:10.1152/ajpheart.00973.2004

Timmermans P. B., Smith R. D. (1994): Angiotensin II receptor subtypes: selective antagonists and functional correlates. Eur. Heart. J. 15, 79-87 
Villanueva I., Piñón M., Quevedo-Corona L., Martínez-Olivares R., Racotta R. (2003): Epinephrine and dopamine colocalization with norepinephrine in various peripheral tissues: guanethidine effects. Life Sci. 13, 1645-1653 doi:10.1016/S0024-3205(03)00491-0

Wang M., Zhang J., Spinetti G., Jiang L. Q., Monticone R., Zhao D., Cheng L., Krawczyk M., Talan M., Pintus G., Lakatta E. G. (2005): Angiotensin II activates matrix metalloproteinase type II and mimics age-associated carotid arterial remodeling in young rats. Am. J. Pathol. 167, 1429-1442 doi:10.1016/S0002-9440(10)61229-1

Wu T. C., Chen Y. H., Leu H. B., Chen Y. L., Lin F. Y., Lin S. J., Chen J. W. (2007): Carvedilol, a pharmacological antioxidant, inhibits neointimal matrix metalloproteinase-2 and -9 in experimental atherosclerosis. Free Radic. Biol. Med. 11, $1508-1522$ doi:10.1016/j.freeradbiomed.2007.08.010

Xie Z., Singh M., Singh K. (2004): Differential regulation of matrix metalloproteinase-2 and -9 expression and activity in adult rat cardiac fibroblasts in response to interleukin-1beta. J. Biol. Chem. 279, 39513-39519 doi:10.1074/jbc.M405844200

Yang E. V., Sood A. K., Chen M., Li Y., Eubank T. D., Marsh C. B., Jewell S., Flavahan N. A., Morrison C., Yeh P. E., Lemeshow S., Glaser R. (2006): Norepinephrine up-regulates the expression of vascular endothelial growth factor, matrix metalloproteinase (MMP)-2, and MMP-9 in nasopharyngeal carcinoma tumor cells. Cancer Res. 21, 10357-10364

doi:10.1158/0008-5472.CAN-06-2496

Received: October 26, 2010

Final version accepted: April 27, 2011 